\title{
OLD MATERIALS - NEW CAPABILITIES: LATTICE MATERIALS IN STRUCTURAL MECHANICS
}

\author{
Marek Augustyniak \\ Gdańsk University of Technology, Department of Technical Physics and Applied Mathematics, Gdańsk, Poland and \\ DES ART Ltd., Gdynia, Poland; e-mail: maugustyniak@mif.pg.gda.pl
}

Lattice materials (LM) are a novel concept stemming from the combination of crystallography and structural optimisation algorithms. Their practical applications have become real with the advent of versatile additive layer manufacturing (ALM) techniques and the development of dedicated CAD/CAE tools. This work critically reviews one of the major claims concerning LMs, namely their excellent stiffness-to-weight performance. First, a brief literature review of spatially uniform LMs is presented, focusing on specific strength of standard engineering materials as compared with novel structures. An original modelling and optimisation is carried out on a flat panel subject to combined shear and bending load. The calculated generalised specific stiffness is compared against reference values obtained for a uniform panel and the panel subjected to topological optimisation. The monomaterial, a spatially repetitive solution turns out to be poorly suited for stiff, lightweight designs, because of suboptimal material distribution. Spatially non-uniform and locally size-optimised structures perform better. However, its advantage over manufacturable, topologically-optimised conventional designs can at best be marginal $(<10 \%)$. Cubic-cell lattices cannot replace conventional bulk materials in the typical engineering use. The multi-cell-type and multi-material lattice structures, albeit beyond the scope of this article, are more promising from the point of view of mechanical properties. The possibility of approaching the linear scaling reported in the recent litterature can make them an attractive option in ultra-low weight designs.

Keywords: lattice materials, additive layer manufacturing, specific stiffness, topological optimisation

\section{Introduction}

\subsection{Definition and classification}

The Lattice Material is a structure generated with the aid of Additive Layer Manufacturing consisting of branched beams usually forming repetitive primitive cells having internal structures derived from crystallography (Face-Centered Cubic, Diamond-like, etc.). The edge of a typical cell does not exceed $10 \mathrm{~mm}$, and the beams cross section tends to be of the order of a 1/10 up to $1 \mathrm{~mm}$ square. Smaller diameters are difficult to manufacture, except for very sophisticated low-series techniques (Bauer et al., 2016).

LMs feature an open structure, and their fabrication method (ALM) naturally allows free modulation of the material effective density.

A rigorous classification of LM (also known as: periodic cellular solids, metamaterials, periodic trusses) is difficult, although claimed by Zok et al. (2016) to be possible. Several criteria have to be employed, including (but not limited to):

- the material used to form beams: polymer/metal

- the external shape of the primitive cell: hex/wedge/tetra, or compound (more than one cell type) 
- the internal "crystallographic" layout: BCC, FCC, diamond, Kelvin-cell, etc.

- the fabrication technology: SLS (Selective Laser Sintering) or FDM (Fused Deposition Modelling).

The spatial, long-range configuration of LM introduces further classes:

- fully uniform (cells are reproduced 1:1, and their properties are quasi-isotropic)

- uniform-directional (cells are reproduced 1:1, but their internal structure produces long-range anisotropy)

- beam-optimised (each beam can have a different diameter).

Table 1 represents schematically the mentioned spatial configurations, adding a "blended" structure in which some design regions are solid while some others created from the lattice structure. Both the diameter-optimised and blended designs require a dedicated calculation algorithm (size + topology optimisation). Their generation requires a case-by-case approach tailored for a given component shape and loading conditions.

Table 1. Basic options for the cubic cell Lattice Material spatial layout

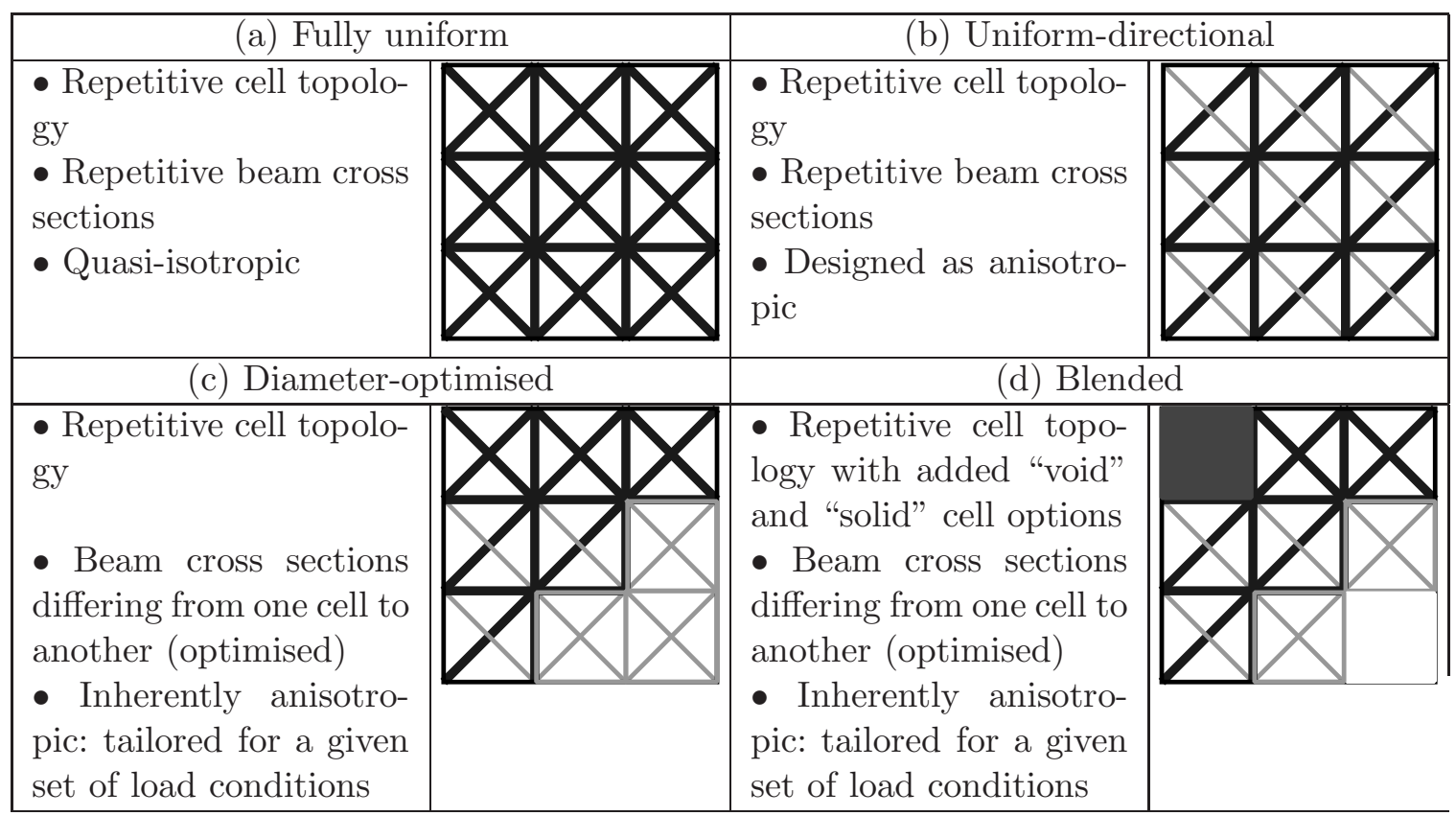

The multi-cell-type and multi-material lattice structures (with further ramifications, as shown in Table 1) remain largely unexplored. This stems from the difficulties in their optimal design and still very limited access to adequate manufacturing technologies. These solutions, promising as they seem, will not be discussed in this paper.

\subsection{Lattice material properties and range of potential applications}

LMs are remarkable for their potential multi-functionality, namely the combination of tuneable or even programmable mechanical, thermal, mass-transport and electromagnetic properties. The word "potential" has to be emphasised, because apart from decorative objects, no inherently "lattice-based" technical designs have so far emerged on the market.

Several authors have studied the sensitivity analysis of various thermomechanical properties of LMs including: static stiffness and strength, energy absorption capacity, vibration properties, effective Poisson's ratio, effective thermal dilatation coefficient and the active surface. In many 
cases, the main stake is mass reduction, or more precisely, optimisation of specific stiffness, specific energy absorption (SEA) or other mentioned parameters.

Some authors have been systematically exploring the design space of varying cell configurations and sizes. Mazur et al. (2016) focused on strength and stiffness of Ti-6Al-4V lattices. Junyi and Balint (2016) added dispersion properties to monitored variables. Lopatin et al. (2017) studied both the intensive (cell structure) and extensive (plate dimensions) parameters in search for the maximum critical buckling load.

Messner (2016) came up with a complete algorithm for generating homogeneous lattice microstructures optimisable from the point of view of the stiffness-to-density ratio. He took the degree of anisotropy into account as well.

The static specific stiffness of LMs has been explored in the context of its:

- maximisation (Messner, 2016; Bauer et al.,2016)

- tuning (for reproduction of human bone stiffness) (Serra-Garcia et al., 2016)

- minimisation (supercompressibility) (Zhu et al., 2015; Jiang and Wang, 2016).

This parameter will be the main focus of this study.

Lattice-type materials have also been discussed in the context of auxetics, i.e. structures with negative Poisson's ratio.

Controlled buckling behaviour, usually studied for fail-safe design or maximisation of energy absorption has been put forward by Lopatin et al. (2017), Paulose et al. (2015), Yin et al. (2017), Hawreliak et al. (2016). Vibration characteristics (selective damping) have been studied by Bacigalupo et al. (2015), Srivastava (2016) and Pasternak et al. (2016).

Thermal and thermo-mechanical properties remain a prominent area of research. Especially, thermal dilatation tuning (e.g. zero CTE for elimination of thermal stresses) has been reported in (Pasternak et al., 2016; Xu and Pasini, 2016; Wang et al., 2016; Toropova and Steeves, 2016). Maximisation of the effective themal conductivity (heat sinks) appeared in works by Wadley and Queheillalt (2007), Kumar et al. (2009), Tian et al. (2007). The list is not exhaustive. Notably, there have been attempts to characterise lattice material durability (Hawreliak et al., 2016), mass-transport properties for possible application in electrodes (Bauer et al., 2016; Zhu et al., 2015). A high surface-to-mass ratio allows the development of more efficient energy-storage structures (Zhu et al., 2015; Sullivan et al., 2016). Last but not least, a still active major research area involves interaction of electromagnetic waves with periodic structures (meta-materials) (Srivastava, 2016 and many others).

\subsection{Goal of this study}

Among several possible functions of LMs, one of the fundamental mechanical properties, namely the stiffness-to-density ratio, is selected for close examination. Several scientific papers hint on the outstanding specific stiffness (and alternatively specific strength) of LMs:

- Mazur et al. (2016) - "One can reach properties beyond the capacity of solid material"

- Yin et al. (2017) - "Hybrid designs can populate vacant regions in mechanical property charts"

- Messner (2016) - "Previous work demonstrates that lattice materials have excellent stiffness- and strength-to-weight scaling, outperforming natural materials (...) making these structures efficient for lightweight structural applications" (quoted after Zheng et al., 2014).

In the above statements it is implied that the specific stiffness of LMs can be significantly maximised by appropriate selection of their internal layout. These claims are even more pronounced in informal reports: 
- Dias (2015) - "LMs also have desirable weight characteristics, and are used as an approach to target weight reduction" (this feature is listed in the third place, after "better performance for stability and larger surface area")

- Krassenstein and Lyles (2014) - "As we know, lattice structures have been shown to increase strength while reducing weight (...) Without a doubt, there could be hundreds or even thousands of uses for lattices such as this for designing and engineering a whole range of products and architectural designs".

However, there are some recent papers (e.g. Zok et al., 2016) where the above claims are put at doubt.

The community of structural mechanics indeed awaits novel stiff and light materials. The red contour, sketched by the author on one of the Ashby-plots (Fig. 1, taken from Ashby and Cebon, 1993), encompasses the area of highly desirable specific stiffness levels with 4-sub-areas corresponding to:

A: structures with a relatively high absolute stiffness, as light as possible, dedicated to a broad engineering use

B: specialised low and ultra-low density structures exhibiting as little stiffness loss as possible

C: hybrid area between A and B

D: most probably infeasible materials.

Another simplified criterion can be set at a straight line corresponding to a constant $E^{1 / 2} / \rho=$ 1/100, with $E$ expressed in GPa and $\rho$ expressed in $\mathrm{kg} / \mathrm{m}^{3}$.

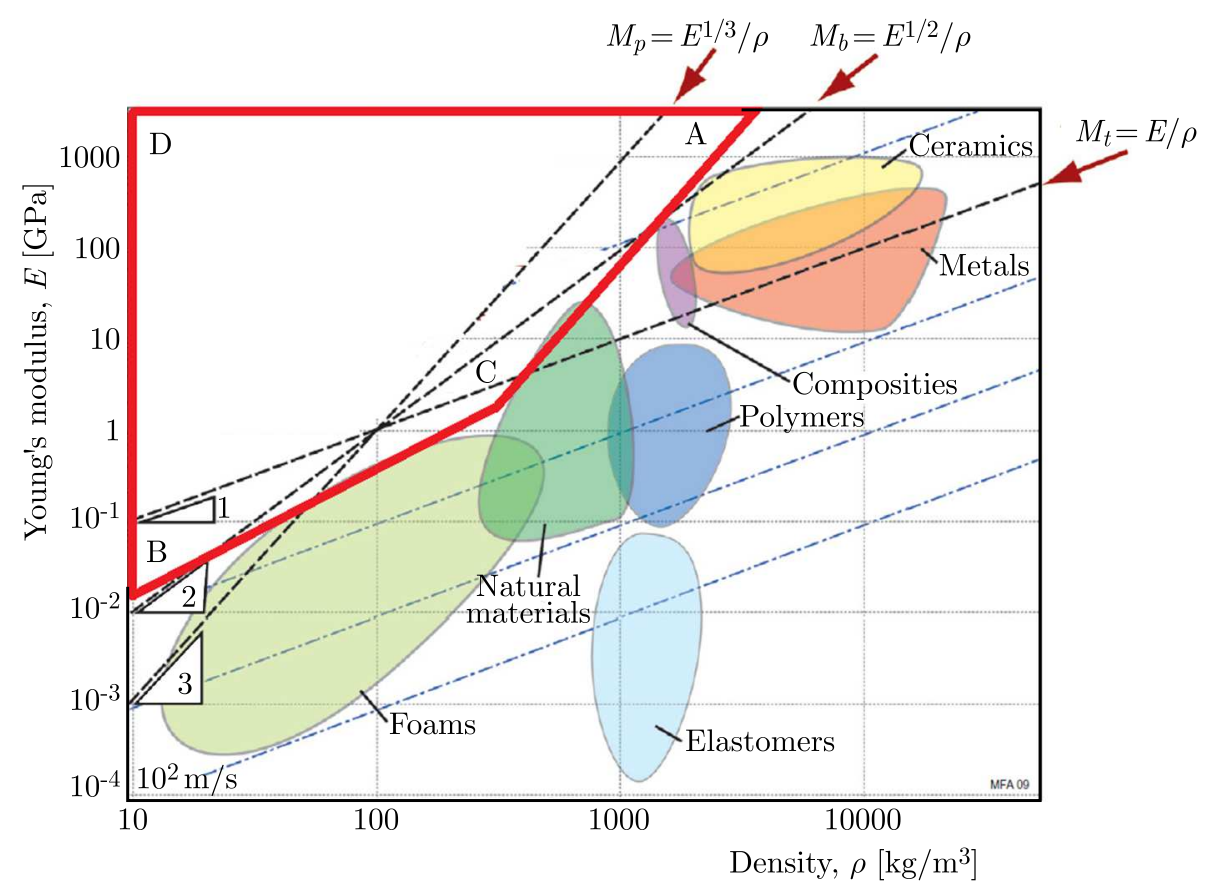

Fig. 1. Material selection chart (Ashby and Cebon, 1993) with sub-areas marked by the author of this article

The key question is: can LMs perform better, at least in terms of increased specific stiffness, than the existing materials, either homogeneous or composite? The areas of the potential widespread engineering use (A and $\mathrm{C}$ in Fig. 1) are addressed in this paper. 
A brief literature review is carried out first in order to quantify the best solutions hitherto found.

Apart from the literature review, the original contribution of this study consists in the design and numerical optimisation of a series globally and locally optimised lattice structures in search for their actual specific stiffness.

In particular, the sensitivity analysis is carried out on a panel of constant external dimensions and varied internal LM configuration. The finite element computations are performed using OptiStruct 14.0 software by Altair. The modelling is supposed to compare the "latticed" design as compared to the best conventional topologically optimised design.

\section{Uniform lattice materials: specific stiffness and strength}

Several authors, as shown in the previous Section, have claimed that LMs are very promising in terms of pushing the limits of specific stiffness and strength. It is relevant to quantify and verify the obtained results by comparing them to the representatives of various material families.

At the beginning, it is necessary to note that in the traditional structural design more than one "specific stiffness" can be defined for a given material (for demonstration, see Ashby and Cebon, 1993). Notably, $E / \rho$ is recommended for pure 1D loading (as in struts, stretched cables), $E^{1 / 2} / \rho$ is adequate for beams carrying bending loads, $E^{1 / 3} / \rho$ characterizes the shells/plates/panels.

Consequently, if both the stiffness and density decrease proportionally (e.g. both by a factor of 10 typical for LMs as compared with the bulk material), the parameters: $E^{1 / 2} / \rho$ and $E^{1 / 3} / \rho$, increase, which is beneficial for lightweight design of beam/plate structures. In the further discussion, special emphasis is put on the "beam-optimised" specific stiffness.

Table 2 puts together selected typical data from metal, polymer and ceramic engineering materials. As expected, the costly carbon-fibre composite turns out to be the best. However, cheap and widely accessible wood comes the second, being particularly well suited for beamoptimised designs. Young's modulus $E$ is here expressed in GPa and $\rho$ expressed in $\mathrm{kg} / \mathrm{m}^{3}$. These units shall be kept further on in this study.

Table 2. Juxtaposition of specific strengths for standard engineering materials (source: Cambridge, 2003)

\begin{tabular}{|l|c|c|c|c|c|}
\hline \multicolumn{1}{|c|}{ Material } & $\begin{array}{c}E \\
{[\mathrm{GPa}]}\end{array}$ & $\begin{array}{c}\rho \\
{\left[\mathrm{kg} / \mathrm{m}^{3}\right]}\end{array}$ & $\begin{array}{c}E / \rho \\
(\text { pure stretch })\end{array}$ & $\begin{array}{c}E^{1 / 2} / \rho \\
(\text { beam })\end{array}$ & $\begin{array}{c}E^{1 / 3} / \rho \\
(\text { plate })\end{array}$ \\
\hline \hline $\begin{array}{l}\text { Flex. polymer } \\
\text { foam (VLD) }\end{array}$ & 0.001 & 35 & $3 \mathrm{E}-5$ & $9 \mathrm{E}-4$ & $3 \mathrm{E}-3$ \\
\hline Polypropylene & 1.2 & 900 & $1 \mathrm{E}-3$ & $1 \mathrm{E}-3$ & $1 \mathrm{E}-3$ \\
\hline WC & 650 & 15600 & $4 \mathrm{E}-2$ & $2 \mathrm{E}-3$ & $6 \mathrm{E}-4$ \\
\hline Plain steel & 200 & 7850 & $3 \mathrm{E}-2$ & $2 \mathrm{E}-3$ & $7 \mathrm{E}-4$ \\
\hline $\begin{array}{l}\text { Rigid polymer } \\
\text { foam (HD) }\end{array}$ & 0.3 & 300 & $1 \mathrm{E}-3$ & $2 \mathrm{E}-3$ & $2 \mathrm{E}-3$ \\
\hline Alu & 75 & 2700 & $3 \mathrm{E}-2$ & $3 \mathrm{E}-3$ & $2 \mathrm{E}-3$ \\
\hline $\begin{array}{l}\text { Typical wood } \\
\text { (longitudinal) }\end{array}$ & 12 & 700 & $2 \mathrm{E}-2$ & $5 \mathrm{E}-3$ & $3 \mathrm{E}-3$ \\
\hline CFRP & 120 & 1500 & $8 \mathrm{E}-2$ & $7 \mathrm{E}-3$ & $3 \mathrm{E}-3$ \\
\hline
\end{tabular}

What is the performance of LMs in this aspect? Table 3 and corresponding Fig. 2 (data taken from Mazur et al., 2016) shows that one the best type of cubic basic cell behaves on average 2 times better than the bulk base material and slightly outperforms pure aluminium. On the 
other hand, the worst case exhibits properties equal to those of polypropylene, which is a very poor outcome for a specialised Ti-Al-V alloy. In terms of beam-optimised specific stiffness, the best LM shows roughly a twofold superiority over plain steel while its absolute stiffness is lower by a factor of 200. As long as such a low stiffness is acceptable in a given design, LM can thus be a valuable alternative to conventional materials.

Table 3. Juxtaposition of specific stiffnesses of the best/worst lattice materials (Mazur et al., 2016)

\begin{tabular}{|l|c|c|c|c|c|}
\hline \multicolumn{1}{|c|}{ Material } & $\begin{array}{c}E \\
{[\mathrm{GPa}]}\end{array}$ & $\begin{array}{c}\rho \\
{\left[\mathrm{kg} / \mathrm{m}^{3}\right]}\end{array}$ & $\begin{array}{c}E / \rho \\
(\text { pure stretch })\end{array}$ & $\begin{array}{c}E^{1 / 2} / \rho \\
(\text { beam })\end{array}$ & $\begin{array}{c}E^{1 / 3} / \rho \\
\text { (plate) }\end{array}$ \\
\hline \hline Ti-6Al-4V bulk & 115 & 4430 & $3 \mathrm{E}-2$ & $2 \mathrm{E}-3$ & $1 \mathrm{E}-3$ \\
\hline $\begin{array}{l}\text { Ti-6Al-4V LM } \\
(1 \% E, 6 \% \rho), \\
\text { FCCZ-S3 (best) }\end{array}$ & 1.15 & 266 & $4 \mathrm{E}-3$ & $4 \mathrm{E}-3$ & $4 \mathrm{E}-3$ \\
\hline $\begin{array}{l}\text { Ti-6Al-4V LM } \\
(1 \% E, 24 \% \rho), \\
\text { FBCCZ0-S2 (worst) }\end{array}$ & 1.15 & 1063 & $1 \mathrm{E}-3$ & $1 \mathrm{E}-3$ & $1 \mathrm{E}-3$ \\
\hline
\end{tabular}

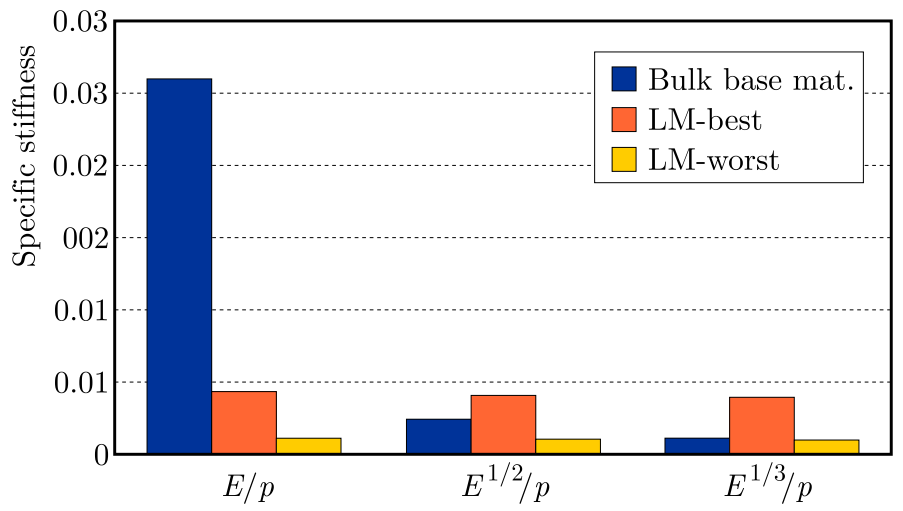

Fig. 2. Juxtaposition of specific stiffnesses of the best/worst lattice materials according to (Mazur et al., 2016)

The recent work by Zheng et al. (2014) has revealed a new solution based on lattices made of hollow tubes with average $E / \rho$ of $3 \mathrm{E}-3$ and outstandingly high $E^{1 / 2} / \rho$ of $3 \mathrm{E}-2$ (with $E$ expressed in GPa and $\rho$ in $\mathrm{kg} / \mathrm{m}^{3}$ ). However, they applied a special octet-truss, a non-cubic structure, and focused on ultra-low density materials which are outside the scope of this research.

It is notable that only few quantitative assessments of specific stiffness (or alternatively specific strength) can be found in literature. Some reports, especially informal ones, are simply misleading. For example, a Purdue researcher working for a commercial 3D printing company (Krassenstein and Lyles, 2014) claims that his "incredible" Kelvin-cell-based aluminium cube supports about 100000 times its weight. The number can be true, but when closely examined, it turns out to be no better than the natural performance of the bulk aluminium for the same cube dimensions.

Some authors hint on scaling the LM structures down as a method for increasing their stiffness and strength. Jiang and Qang (2016) declares that under large-strain tension, their moduli follow a linear scaling relationship with their densities regardless of architecture types, which is in sharp contrast to the architecture-dependent modulus power-law of the existing engineering materials. Mazur et al. (2016) and Messner (2016) reported a convergence of mechanical properties with the increasing number of unit cells. The quantitative data produced by Bauer et al. 
(2016) (concerning strength only) did not, however, provide convincing proofs of the superiority over the bulk base material. They aim at exploiting material strengthening size effects and achieving strength-to-density ratios of "nanolattices" 6 times those of other reported microlattices. However, their 1 micrometer-long struts with 200-nanometer diameters made of pyrolysed carbon exhibit compressive specific strength inferior to those of diamond $\left(1.2 \mathrm{GPa}\right.$ at $600 \mathrm{~kg} / \mathrm{m}^{3}$ as compared with $>110 \mathrm{GPa}$ at $3500 \mathrm{~kg} / \mathrm{m}^{3}$ ). Thus the carbon-based microlattice is $20 \times$ less stress-resistant (and probably just as less stiff) than the diamond while much more expensive to manufacture.

Again, it has to be emphasised, that the best structurally optimised LMs can be beneficial uniquely in very-low-weight designs, where their stiffness can decrease proportionally to the decreasing density, as compared to much more unfavourable scaling relationship of stochastic foams.

Judging from the literature data, the spatially uniform Lattice Materials, regardless of the scale of their structure (meso/micro/nano), are not significantly superior to the existing non-lattice materials in terms of specific stiffness as long as relatively stiff structures (e.g. $E>100 \mathrm{MPa})$ are required. Even if some carefully selected structures show an increased $E^{1 / 2} / \rho$ over the bulk base material, they do not penetrate deep into the desirable zones on the Ashby plot. It is partly reflected in the conclusion of the paper by Zok et al. (2016): "numerous trusses that have been studied in recent years do not appear to be particularly well-suited for use as stiff and strong lightweight structures on their own".

\section{Single-material lattices vs topologically optimised base material: original modelling}

The literature review suggests that spatially uniform Lattice Materials are not likely to achieve outstanding specific stiffness. However, spatial variation of the cell type and beam diameters is intuitionally beneficial, and has been suggested by some authors (e.g. Messner, 2016). Some simple solutions can be directly borrowed from the classic design rules, e.g. adding of solid skins atop of LM panels in order to increase their bending stiffness. On the other hand, Additive Layer Manufacturing (ALM), costly as it is, offers in exchange almost unlimited freedom in structural shaping, so it is natural to look for arbitrarily "free" non-standard designs often inspired by biological systems. Automatic optimisation procedures including topology and size optimisation have reached maturity, and now are widely used in industry as witnessed by the author in his engineering practice. It is possible that both these approaches are applicable to design of light and stiff LMs. However, such a hybrid methodology has not been widely used yet. The only quantitative data of the beneficial LM effect comes from commercial presentation (Dias, 2015), where a $12 \%$ increase in the of stiffness of an actual automotive part is reported as compared with the topologically optimised manufacturable design without a lattice structure. More data is definitely needed.

In this paper, the author generates a relatively simple model which is useful in studying the impact of Topological Optimisation (TO) and LM techniques, separately or in combination.

The model (Fig. 3) involves a $2 \mathrm{~mm}$ thick plate made of Nylon12PA, typical for 3D-printing applications, dimensioned $240 \times 32 \mathrm{~mm}$. The plate is supported along its shorter edges and is loaded with a field of uniform concentrated forces at its geometrical centre. The supports are designed so that the mechanism is avoided, but there are no singularities due to overconstraining at the same time.

A series of FEA calculations have been carried out in order to examine the variation of the specific stiffness. No stress constraints have been applied. The studied solutions are presented in Table 4. 


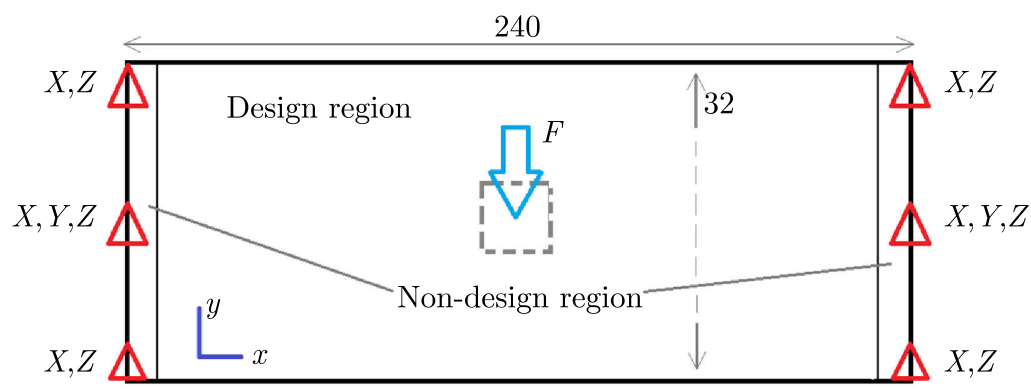

Fig. 3. A schematic view of the design space for specific strength analysis

Table 4. List of structural variants selected for specific strength comparison

\begin{tabular}{|l|l|}
\hline 100 & Starting design: a full plate \\
\hline 200 & $\begin{array}{l}\text { Topological optimisation with a volume constraint set at } 30 \% \text { of the initial } \\
\text { design region volume and objective defined as compliance minimisation }\end{array}$ \\
\hline 400 & $\begin{array}{l}\text { A series of LM designs with the total plate volume uniformly transformed into } \\
\text { lattice; the beam diameter (equal for every element) is made variable }\end{array}$ \\
\hline 600 & Hybrid, "blended" solution \\
\hline
\end{tabular}

The analyses refer to the case-specific "generalised" metrics of compliance and stiffness, typical for numerical studies of engineering components. The simplest definition of a generalised compliance is a vector product $\mathbf{F} \cdot \mathbf{u}$ summed over all nodes, where $\mathbf{F}$ is the applied force vector, and $\mathbf{u}$ - vector of resultant displacements. Such a function represents equally strain energy of the component.

Additionally, it is practical to replace the density with the component mass, so that the generalised specific stiffness (GSS) is equal to the generalised stiffness/component mass or alternatively: 1/(generalised compliance-component mass). So defined GSS will be the key parameter in the following discussion.

\subsection{Design 100 - starting design, full plate}

Standard linear-static analysis has been performed. The displacements plot is presented in Fig. 4.

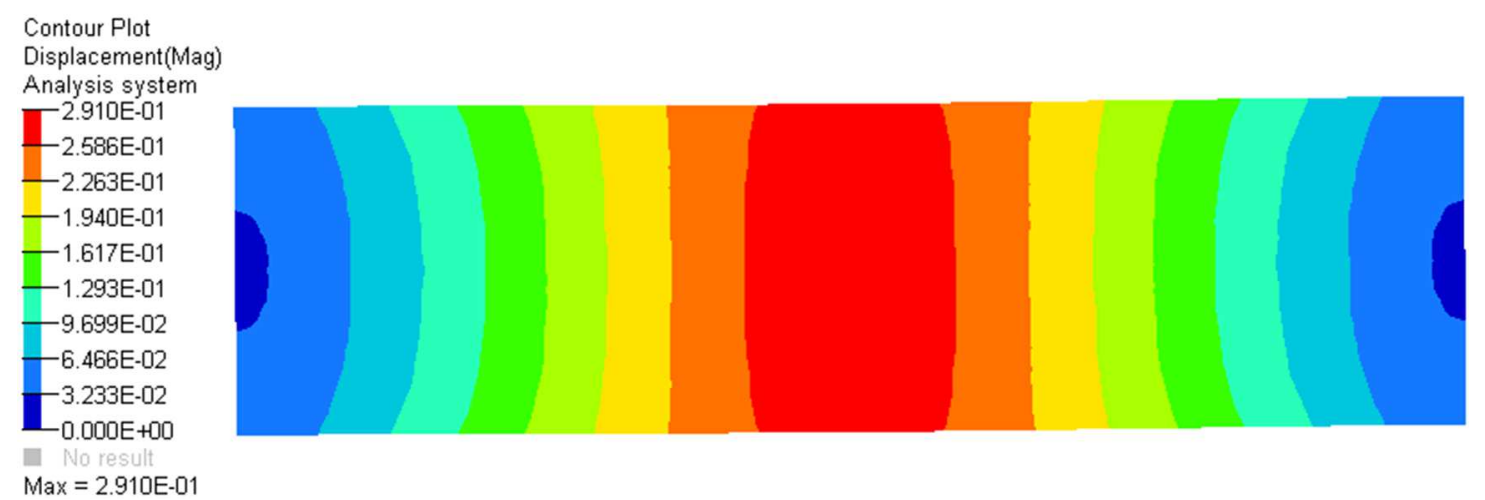

Fig. 4. The magnitude of displacement for the starting, full-plate design (100)

The bulk plate weighs $6.8 \mathrm{~g}$. The behaviour of the plate, both in terms of deflection and stress distribution (not shown here), is consistent with the expectations derived from the classical beam theory. The Generalised Specific Stiffness (GSS) has been calculated, and set as a $100 \%$ reference for the subsequent options. The numerical data are presented at the end of the article, in Table 6 . 


\subsection{Design 200 - topological optimisation (TO)}

The design space has been subjected to a typical topological optimisation procedure based on the SIMP (Solid Isotropic Material with Penalisation) algorithm. A mass constraint has been set so as to finally reduce the mass of the design region by $30 \%$. The TO algorithm converged after 20 iterations, producing pseudo-density distribution as in Fig. 5. The red areas represent the material which needs to be kept while dark blue zones indicate the redundant material to be removed. The uneven contour boundaries can be manually or automatically smoothened out. An automatic, rough, yet meaningful interpretation of the final design is shown in Fig. 5.

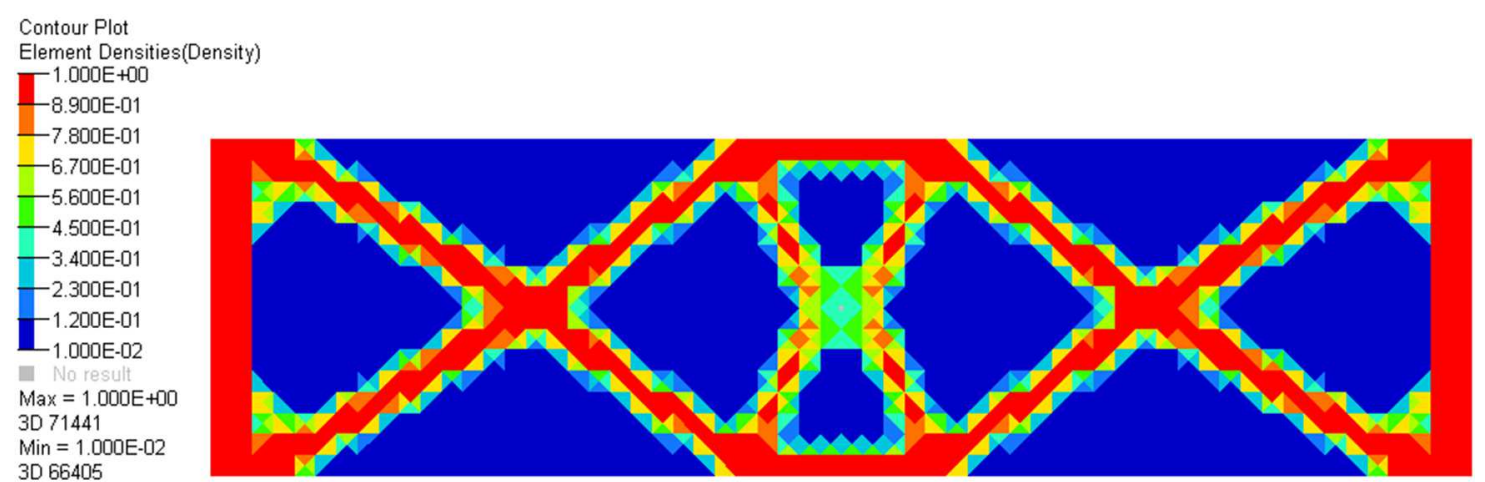

Fig. 5. Element pseudo-density distribution at the final iteration of the topological optimisation process (200)

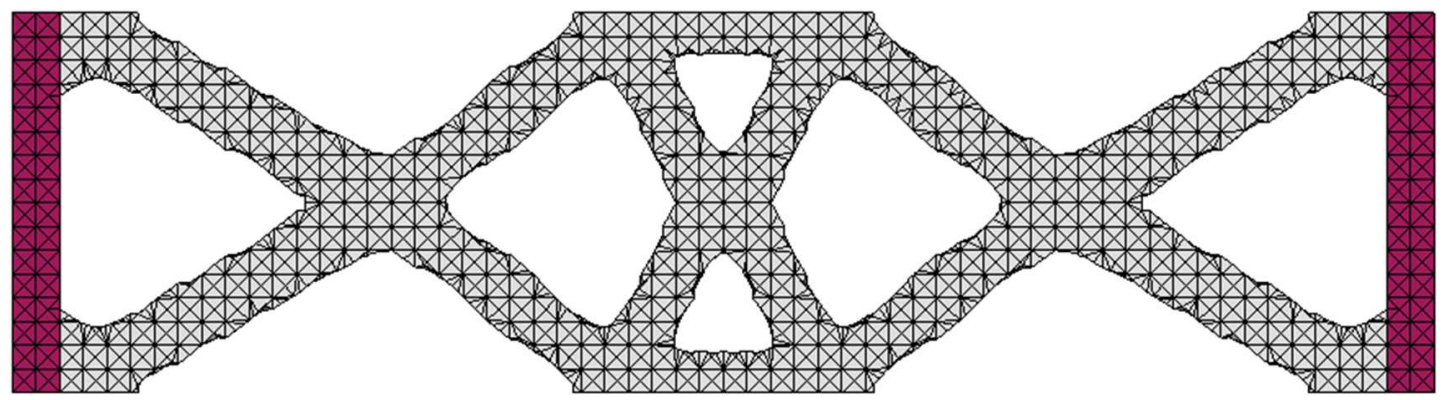

Fig. 6. Rough automatic interpretation of the topologically optimised structure for subsequent reanalysis or manufacturing

The normalised Generalised Specific Stiffness (GSS) for this design is slightly below the reference value and amounts to its $86 \%$. The topological optimisation, it this particular case, does not bring benefits in terms of specific stiffness. However, it allows identifying weakly stressed areas and results in mass reduction which is an obvious advantage in industrial applications as long as constraints on allowable displacements are not violated.

\subsection{Design 400 - uniform lattice with variable beam diameter}

The entire plate has been transformed into a LM, except for its constrained edge bounds. The elementary cell dimensions are directly inherited from the finite element mesh. The beams are assigned with an arbitrary, constant diameter with the starting value of $0.1 \mathrm{~mm}$. 


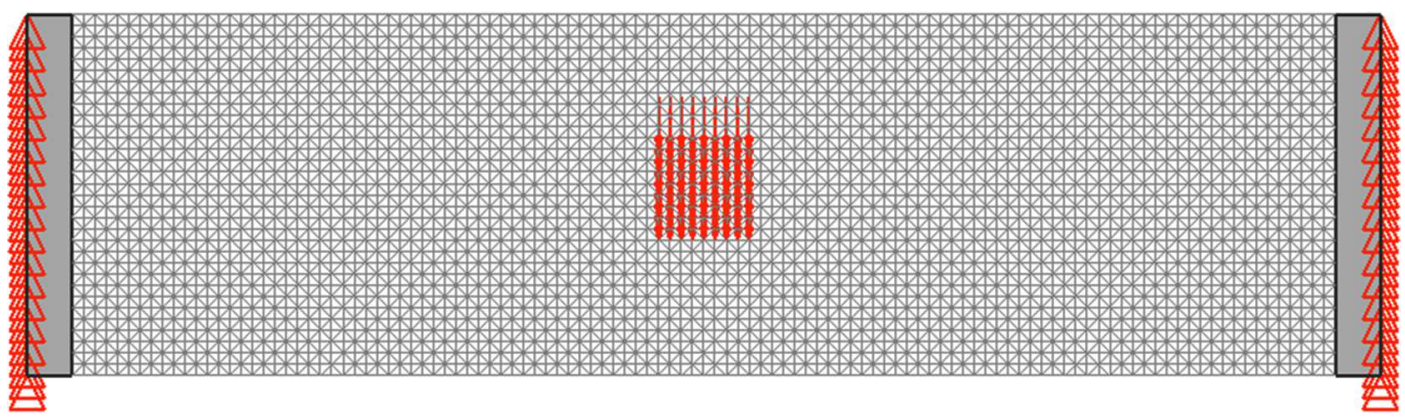

Fig. 7. The plate transformed into an uniform lattice structure (400)

Table 5. Influence of variation of the beam diameter on the generalised specific stiffness

\begin{tabular}{|l|c|c|}
\hline & Relative density [\%] & GSS \\
\hline \hline 400 LM-uniform diameter $0.1 \mathrm{~mm}$ & 12 & 0.0009 \\
\hline 400 LM-uniform diameter $0.2 \mathrm{~mm}$ & 26 & 0.0015 \\
\hline 400 LM-uniform diameter $0.3 \mathrm{~mm}$ & 51 & 0.0018 \\
\hline 100 bulk & 100 & 0.0069 \\
\hline
\end{tabular}

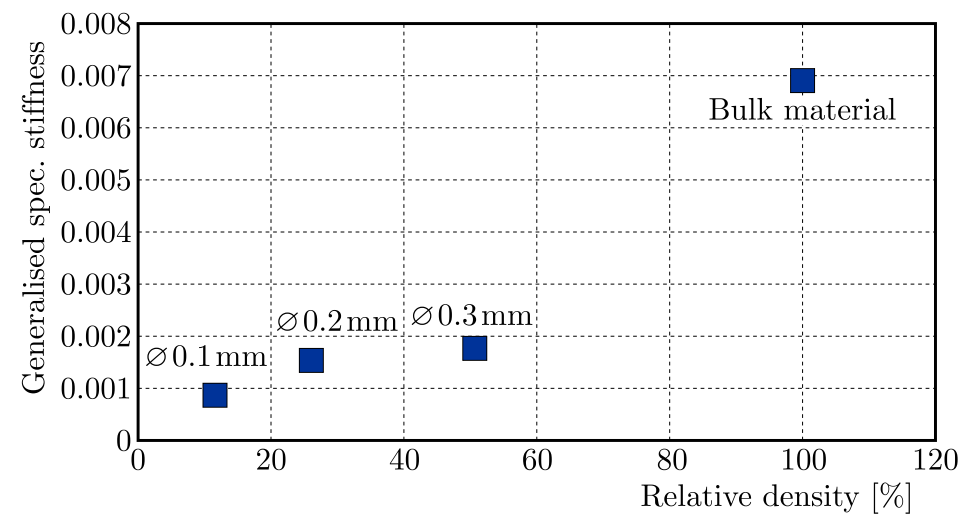

Fig. 8. The influence of variation of the beam diameter on the generalised specific strength: plot related to relative density

The sensitivity analysis is summarised in Table 5 and Fig. 8. Two adverse effects are noticeable:

1) Calculation at $50 \%$ of the relative density is visibly biased. If beam diameters are significant as compared with their lengths, the problem of overlapping at beam joints arises, and the nominal mass is overestimated. An actual 3D-printed object shall be lighter than the sum of masses of beams included in the FE model.

2) The specific stiffness definitely does not improve when replacing the bulk material with a LM. On the contrary, for this design space and set of loads the effect of "latticing" is detrimental. As compared with the starting simple bulk plate, the GSS (for $0.1 \mathrm{~mm}$ beams) drops by a factor of 7 , which entails about a $60 \times$ decrease in the absolute stiffness.

\subsection{Design 600 - hybrid (blended) solution}

This design combines the results from Topology Optimisation with the partial Lattice transformation according to the lower and upper bounds arbitrarily set by the user. Here the bounds are 0.15 and 0.6 , which entails that relative densities below 0.15 are removed (a void remains) while the elements exhibiting densities beyond 0.6 remain as solid. 


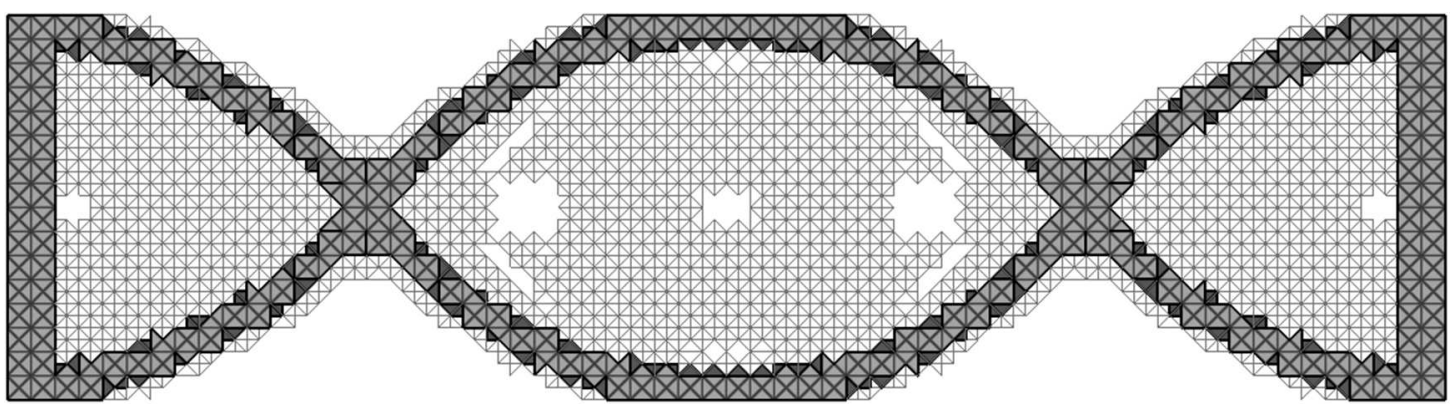

Fig. 9. An example of a "blended" design, combining the TO - resultant core structure and auxiliary lattice

The obtained solution has several remarkable features:

1) The GSS for this solution is very close to the one from pure Topological Optimisation, however it can possibly be increased if beam-by-beam size optimisation is employed.

2) The entire structure is a "composite" with locally varying stiffnesses and densities, although it is actually made of a single base material.

3) The design after smoothening and cleaning up can be manufactured in a single-pass FDM or SLS technology.

4) Although in this hybrid solution the lattice does not play a significant role in terms of stiffness or strength, it can serve as a filler or a supportive structure for additional functionalities (e.g. heat exchange, cabling, sensor arrays, etc.).

5) The similar, automotive structure redesigned with a "blended" technique has been reported to be $\sim 10 \%$ stiffer than the solution by Topological Optimisation only with the imposed manufacturing constraints.

The summary of calculated GSS is presented in Table 6 .

Table 6. Complete comparison of generalised specific sttiffnesses for the studied design options

\begin{tabular}{|l|c|c|c|c|c|}
\hline & $\begin{array}{c}\text { Mass } \\
{[\mathrm{g}]}\end{array}$ & $\begin{array}{c}\text { Generalised } \\
\text { compliance } \\
{[\mathrm{N} \cdot \mathrm{mm}]}\end{array}$ & $\begin{array}{c}\text { Generalised } \\
\text { stiffness }\end{array}$ & $\begin{array}{c}\text { Generalised } \\
\text { spec. stiffness } \\
\text { (GSS })\end{array}$ & $\begin{array}{c}\text { Normalised } \\
\text { GSS [\%] }\end{array}$ \\
\hline \hline 100 Bulk material & 7.3 & 19.8 & 0.05 & 0.0069 & 100 \\
\hline $\begin{array}{l}\text { 200a TO, volumic goal of } \\
\text { design space set to 30\% }\end{array}$ & 2.5 & 67.5 & 0.01 & 0.0059 & 86 \\
\hline $\begin{array}{l}\text { 200b TO, volumic goal of } \\
\text { design space set to 40\% }\end{array}$ & 3.2 & 48.3 & 0.02 & 0.0065 & 93 \\
\hline 400 LM-Uniform diameter 0.1 & 0.84 & 1370 & 0 & 0.0009 & 13 \\
\hline 400 LM-Uniform diameter 0.2 & 1.9 & 343 & 0 & 0.0015 & 22 \\
\hline 400 LM-Uniform diameter 0.3 & 3.7 & 153 & 0.01 & 0.0018 & 26 \\
\hline 600 LM-blended & 2.5 & 67.4 & 0.01 & 0.0059 & 86 \\
\hline
\end{tabular}

\section{Conclusions and perspectives}

The literature review and original modelling focused on the stiffness performance of lattice materials have led to the following conclusions: 
- In the studied case, a simple conversion of the bulk material into a lattice results in a significant decrease of its stiffness; the best uniform LM cases reported in the literature show stiffnesses comparable to that of the bulk base material, however they do never exceed it. The claims of superiority of LMs in terms of light and stiff designs have to be refuted.

- In spite of the literature reports on the beneficial scale effect, one should not expect lattice-related specific strength higher that for the bulk base material, probably even if micrometre-scale elementary cells are produced.

- Some (but not dramatic) improvement of LM-related GSS can be aimed at when performing beam-by-beam size optimisation; such algorithms are already available in scientific and commercial contexts.

- The only convincing report on GSS increase due to application of the LM base on a hybrid (blended) design, in which the topologically optimised "backbone" made of the bulk material plays a dominant role in providing stiffness. In the areas of intermediate resulting densities (30\%-70\%) it is replaced with lattices. Even if such hybrid optimisation is employed, the reported gain is of the order of $10 \%$ as compared with pure topological optimisation with manufacturing constraints. Still the gain requires sophisticated, blended solid/LM designs, based on two-stage optimisation (Topological+Size). The final solution is of the order of magnitude more costly in production than the standard design (bulk material after TO).

- To meet the engineering goals and be economically viable, lattice materials should be:

- structurally non-uniform to exploit the inherent freedom of design provided by ALM,

- multifunctional, similarly to foams (cf. Banhart, 2005).

Stiffness and/or strength can only constitute an auxiliary function in lattice-material designs. The primary function can be thermal (e.g. embedded efficient heat exchange), thermomechanical (e.g. stress-free intermaterial adapters) or electromagnetic (integrated cables, sensors, screening systems).

- LMs do not deliver a break-through in the area of stiff lightweight design. At present, there are only very limited "hi-tech" fields of their application (aerospace, biomedical, military).

The search for light and stiff micro/macro-optimised solution continues. Interestingly, some authors (Zimińska et al., 2016; Dong and Wadley, 2015) hint on CFRP lattices as being capable of crossing the present limits of available specific stiffnesses. LM multi-material composites can possibly fill the gap of the material selection charts. This, however, is a subject for another study.

\section{References}

1. Ashby M., Cebon D., 1993, Materials selection in mechanical design, Journal de Physique IV Colloque, 03, C7, C7-1-C7-9

2. Bacigalupo1 A., Lepidi M., Gnecco G., Gambarotta L., 2015, Optimal design of auxetic hexachiral metamaterials with local resonators, Smart Materials and Structures, 25, 5

3. Banhart J., 2005, Aluminium foams for lighter vehicles, International Journal of Vehicle Design, 37

4. Bauer J., Schroer A., Schwaiger R., Kraft O., 2016, Approaching theoretical strength in glassy carbon nanolattices, Nature Materials, 15, 438-443

5. Cambridge Press, 2003, Materials Data Book, 2003 Edition, Cambridge University Engineering Department 
6. Dias W., 2015, http://insider.altairhyperworks.com/design-and-optimization-of-lattice-structures -for-3d-printing-using-altair-optistruct/

7. Dong L., Wadley H., 2015, Mechanical properties of carbon fiber composite octet-truss lattice structures, Composites Science and Technology, 119, 26-33

8. Hawreliak J.A., Lind J., Maddox B., Barham M., Messner M., Barton N., Jensen B.J., Kumar M., 2016, Dynamic behavior of engineered lattice materials, Nature Scientific Reports, 6 , 28094

9. JiAng Y., WANG Q., 2016, Highly-stretchable 3D-architected mechanical metamaterials, Nature Scientific Report, 6, 34147

10. Junyi L., Balint D.S., 2016, A parametric study of the mechanical and dispersion properties of cubic lattice structures, International Journal of Solids and Structures, 91, 55-71

11. Krassenstein E., Lyles D.P., 2014, https://3dprint.com/29958/3d-printed-aluminum-lattice/

12. Kumar V., Manogharan G., Cormier D.R., 2009, Design of periodic cellular structures for heat exchanger applications, 20th Annual International Solid Freeform Fabrication Symposium

13. Lopatin A.V., Morozov E.V., Shatov A.V., 2017, Buckling of the composite anisogrid lattice plate with clamped edges under shear load, Composite Structures, 159, 72-80

14. Messner M.C., 2016, Optimal lattice-structured materials, Journal of the Mechanics and Physics of Solids, 96, 162-183

15. Mazur M., Leary M., Sun S., VCelka M., Shidid D., Brandtet M., 2016, Deformation and failure behaviour of Ti-6Al-4V lattice structures manufactured by selective laser melting (SLM), The International Journal of Advanced Manufacturing Technology, 84, 5, 1391-1411

16. Pasternak E., Shufrin I., Dyskin A., 2016, Thermal stresses in hybrid materials with auxetic inclusions, Composite Structures, 138, 313-321

17. Paulose J., Meeussen A.S., Vitelli V., 2015, Selective buckling via states of self-stress in topological metamaterials, Proceedings of the National Academy of Sciences, USA, 112, 7639-7644

18. Serra-Garcia M., Lydon J., Daraio C., 2016, Extreme stiffness tunability through the excitation of nonlinear defect modes, Physical Review E, 93

19. SRIVAstava A., 2016, Metamaterial properties of periodic laminates, Journal of the Mechanics and Physics of Solids, 96, 252-263

20. Sullivan K.T., Zhu C., Duoss E.B., Gash A.E., Kolesky D.B., Kuntz J.D., Lewis J.A., SpAdACCINi C.M., 2016, Controlling material reactivity using architecture, Advanced Materials, 28, 1934-1939

21. Tian J., Lu T.J., Hodson H.P., Queheillalt D.T., Wadleyet H.N.G., 2007, Cross flow heat exchange of textile cellular metal core sandwich panels, International Journal of Heat and Mass Transfer, 50, 2521-2536

22. Toropova M.M., Steeves C.A., 2016, Bimaterial lattices as thermal adapters and actuators, Smart Materials and Structures, 25, 11

23. Wadley H.N.G., Queheillalt D.T., 2007, Thermal applications of cellular lattice structures, Materials Science Forum, 539-543, 242-247

24. Wang Q., Jackson J.A., Ge Q., Hopkins J.B., Spadaccini C.M., Fang N.X., 2016, Lightweight mechanical metamaterials with tunable negative thermal expansion, Physical Review Letter, 117

25. Xu H., PAsini D., 2016, Structurally efficient three-dimensional metamaterials with controllable thermal expansion, Nature Scientific Reports, 6, 34924

26. Yin S., Li J., Binghe L., Kangpei M., Huan Y., Nutt S.R., Xu J., 2017, Honeytubes: Hollow lattice truss reinforced honeycombs for crushing protection, Composite Structures, 160, 1147-1154 
27. Zheng X., Lee H., Weisgraber T.H., Shusteff M., DeOtte J., Duoss E.B., Kuntz J.D., Biener M.M., Ge Q., Jackson J.A., Kucheyev S.O., Fang N.X., Spadaccini C.M., 2014, Ultralight, ultrastiff mechanical metamaterials, Science, 344, 1373-1377

28. Zhu C., Han T.Y.-J., Duoss E.B., Golobic A.M., Kuntz J.D., Spadaccini C.M., Worsley M.A., 2015, Highly compressible 3D periodic graphene aerogel microlattices, Nature Communications, 6, 6962

29. Ziminska M., Dunne N., Hamilton A.R., 2016, Porous materials with tunable structure and mechanical properties via templated layer-by-layer assembly, ACS Applied Materials and Interfaces, 8, 34, 21968-21973

30. Zok F.W., Latture R.M., Begley M.R., 2016, Periodic truss structures, Journal of the Mechanics and Physics of Solids, 96, 184-203

Manuscript received April 28, 2017; accepted for print September 13, 2017 